\title{
The implementation of a community-based aerobic walking program for mild to moderate knee osteoarthritis: A knowledge translation randomized controlled trial: Part II: Clinical outcomes
}

\author{
Lucie Brosseau ${ }^{1 *}$, George A Wells ${ }^{2}$, Glen P Kenny ${ }^{3}$, Robert Reid ${ }^{4}$, Andreas Maetzel ${ }^{5}$, Peter Tugwell ${ }^{6}$, \\ Maria Huijbregts ${ }^{7}$, Carolyn McCullough ${ }^{8}$, Gino De Angelis ${ }^{9}$ and Lily Chen ${ }^{10}$
}

\begin{abstract}
Background: Osteoarthritis $(\mathrm{OA})$ is the most common joint disorder in the world, as it is appears to be prevalent among $80 \%$ of individuals over the age of 75 . Although physical activities such as walking have been scientifically proven to improve physical function and arthritic symptoms, individuals with OA tend to adopt a sedentary lifestyle. There is therefore a need to improve knowledge translation in order to influence individuals to adopt effective self-management interventions, such as an adapted walking program.

Methods: A single-blind, randomized control trial was conducted. Subjects $(n=222)$ were randomized to one of three knowledge translation groups: 1) Walking and Behavioural intervention (WB) (18 males, 57 females) which included the supervised community-based aerobic walking program combined with a behavioural intervention and an educational pamphlet on the benefits of walking; 2) Walking intervention (W) (24 males, 57 females) wherein participants only received the supervised community-based aerobic walking program intervention and the educational pamphlet; 3) Self-directed control (C) (32 males, 52 females) wherein participants only received the educational pamphlet. One-way analyses of variance were used to test for differences in quality of life, adherence, confidence, and clinical outcomes among the study groups at each 3 month assessment during the 12-month intervention period and 6-month follow-up period.
\end{abstract}

Results: The clinical and quality of life outcomes improved among participants in each of the three comparative groups. However, there were few statistically significant differences observed for quality of life and clinical outcomes at long-term measurements at 12-months end of intervention and at 6-months post intervention (18-month follow-up). Outcome results varied among the three groups.

Conclusion: The three groups were equivalent when determining the effectiveness of knowledge uptake and improvements in quality of life and other clinical outcomes. OA can be managed through the implementation of a proven effective walking program in existing community-based walking clubs.

Trial registration: Current Controlled Trials IRSCTNO9193542

Keywords: Osteoarthritis, Clinical trial, Walking, Compliance, Adherence, Education, Behavioural intervention, Guidelines implementation, Knowledge translation

\footnotetext{
* Correspondence: lucie.brosseau@uottawa.ca

${ }^{1}$ Public Health, specialization in Epidemiology, University Research Chair, School of Rehabilitation Sciences, University of Ottawa, Ottawa, Canada Full list of author information is available at the end of the article
} 
Osteoarthritis (OA) is one of the most disabling degenerative diseases affecting the elderly [1]. While the reported prevalence of knee OA is approximately 3\% among individuals between the ages of 45 to 54 years, this number rises to $27 \%$ for those between 63 and 69 years, and increases once again to $44 \%$ for persons above the age of 80 years [2]. The critical challenge is to develop physical activity (PA) programs that will encourage individuals with OA to not only to initiate, but also to maintain improvements in exercise behaviour over a long-term period. This study implemented relatively low-cost community-based walking programs at existing walking clubs.

The second part of this manuscript examines the effect of a proven effective walking program based on the Ottawa Panel CPG [3-5] and implemented through the use of a multifaceted KT intervention (Part I) [6]. This portion focuses on the "Outcome evaluation (Clinical outcomes)" phase of the Knowledge Translation Action Cycle (KTAC) $[7,8]$ introduced in part I [6].

\section{Background}

\section{Physical activity and quality of life}

PA can exacerbate age-related decreases in joint health, functional status, and quality of life (QOL) [9-11]. Moreover, the prolonged physical inactivity observed in individuals suffering from arthritis increases their risk for chronic disease such as coronary heart disease, diabetes, hypertension, obesity and osteoporosis [12,13].

A recent study reported that only $27.8 \%$ of individuals with $\mathrm{OA}$ engaged in PA on a regular basis (i.e. 20 minutes a day, 5 days per week, or 30 minutes per day, 3 days per week) [13]. Promotion of PA, especially in a communitybased context, is a priority for health organizations serving the general population $[13,14]$ and is highly recommended for subgroups affected by chronic diseases including OA [15-17].

Various PA programs for OA have shown significant and beneficial effects on QOL at 2 and 3 months [18-24]. However, this effect was not maintained after a period of unsupervised PA [20,21,25]. According to Hurley [26], "exercise benefits people with OA while people are exercising; when they stop exercising the benefits can be maintained for a short time, but the gains are likely to be lost overtime unless individuals are actively encouraged to continue exercising".

\section{Behavioural interventions}

Behavioural interventions have been used with other chronic conditions to improve long-term maintenance of PA regimens with varying success. Patient education, health counselling, goal setting, telephone contacts, exercise logs, social/peer support and positive feedback either alone or in various combinations, have been studied in cardiovascular disease [27-30] and rheumatoid arthritis [31]. As with OA, multifaceted approaches appear to have the greatest impact on long-term adherence, degree of PA performed, and QOL.

The purpose of this randomized controlled trial (RCT) was to compare 1) improvements in quality of life (QOL) (primary outcome) and clinical outcomes such as pain, mobility and endurance; 2) adherence rates; and 3) confidence and self-efficacy after the implementation of a 12-month supervised community-based aerobic walking program (SCAWP) based on the Ottawa Panel clinical practice guidelines (CPG) [3-5] among three knowledge translation (KT) intervention arms. QOL, confidence, and self-efficacy were compared at 12months (end of treatment) and at 18-months (6-months post-intervention). Adherence was compared during the intervention period at 3, 6, 9 and 12- months. Adherence, confidence and self-efficacy results are presented in part I of this manuscript.

\section{Methods \\ Design}

A single blind randomized controlled trail was used for this study. The hypothesis of this study was that a supervised community-based walking program would have a positive impact on participants with mild to moderate OA of the knee, if they walked regularly as stated in the recommendations of the Ottawa Panel [3-5]. This community-based study was approved by the University of Ottawa Research Ethics Board as well as by the City of Ottawa Public Health Research Ethics Board. All participants signed informed consent. Please see part I for more details on the study design [6].

\section{Sample and recruitment}

A total of 222 adults with mild to moderate OA of the knee were recruited from Ottawa, Ontario and Gatineau, Québec, Canada. Please see part I for inclusion/exclusion criteria [6].

\section{Intervention}

Participants were randomly assigned to one of the three $\mathrm{KT}$ intervention groups using central randomization [32] and computer generated numbers [33]: 1) Walking and Behavioural intervention (WB) ( implementation strategy) (18 males, 57 females) which included the SCAWP with a behavioural intervention and an educational pamphlet on the benefits of walking; 2) Walking intervention (W) (implementation strategy) (24 males, 57 females) wherein participants only received the SCAWP intervention and the educational pamphlet; 3) Self-directed control (C) (dissemination strategy) (32 males, 52 females) wherein participants only received the educational pamphlet. All 3 groups were provided 
with pedometers and log books to be completed to measure physical performance and additional PA aside from the walking sessions. The $\mathrm{KT}$ and dissemination strategies were implemented over a 12-month duration and participants were assessed for additional 6 months (15 and 18-month assessments). A detailed description of each intervention can be found in part I of this manuscript.

\section{Data collection}

Participants were assessed by an independent evaluator at baseline and at each 3 month interval (months 3, 6, 9, 12) during the intervention period. Participants were then assessed at 3 and 6 months post-intervention during the follow-up period (months 15 and 18). Participants were asked to complete a collection of validated questionnaires as well as perform physical tests as each assessment.

\section{Clinical outcome measures Health-related quality of life}

The Arthritis Impact Measurement Scale (AIMS2) and the Short Form (SF-36) questionnaires were administered during patient evaluations. The scientific literature in rheumatology recommends that researchers use the AIMS2 and SF-36 concurrently, as they complement one another [34-36]. In support of their complementary roles, the detection of clinically important changes is enhanced through the use of a disease specific instrument (AIMS2), while a generic measure (SF-36) properly distinguishes different levels of self-reported general health statuses and co-morbidities not necessarily related to OA.

The SF-36 is the most widely used and extensively validated generic measure of health-related QOL [36-38]. The SF-36, which consists of 8 subscales and 36 questions, is a reliable and valid scale for several medical conditions, including rheumatic diseases [35,36,39-41]. Internal consistency via Cronbach's alpha ranged between 0.74 to 0.96 over the different sections [40]. Testretest intra-class correlation coefficients range from 0.75 to 0.93 [41]. Validity testing revealed that the SF-36 correlates moderately with several other measures related to disease activity, pain, depression and QOL [36,41]. The SF-36 was used to report QOL in several RCTs involving various PA programs designed for individuals with OA $[19,21,24]$. The responsiveness of this instrument was demonstrated in these studies by appropriate and expected changes in mean scores according to age, sex, marital status, severity of OA, and presence or absence of co morbid conditions. Mean scores also changed appropriately over time in response to the interventions. The aggregated SF-36 components are scored in which higher scores represented better health-related quality of life [42].

The Arthritis Impact Measurement Scale (AIMS2) is a disease-specific QOL scale [36] that consists of 101 items and includes various subscale scores [42]. The AIMS2 walking and bending sub-scale was considered as the primary outcome. All other AIMS2 sub-scales were used as secondary outcomes, since the use of the global scale could not be aggregated. The global AIMS2 scales are scored in which a low value indicated a higher health status $[43,44]$.This QOL instrument has been used in several walking program studies that included subjects with OA $[18,22,45]$. AIMS2 has been shown to possess good internal consistency (alpha coefficients ranging from 0.72 to 0.91 ) and good test-retest results (Intraclass correlation coefficients ranging from 0.78 to 0.94). The construct and criterion validity have also been examined. Standardized response means for changes in AIMS2 scores over 3 months ranged from 0.36 to 0.80 $[36,44]$.

Functional status was measured using the Western Ontario and McMaster Universities Osteoarthritis Index (WOMAC) (Bellamy and Buchanan, 1986). WOMAC is a self-administered multidimensional index containing dimensions for pain, stiffness and function. This fivepoint instrument has been shown to be valid and reliable in individuals with hip or knee rheumatic conditions, and is sensitive to change over time. The WOMAC questionnaire [45] has been adapted for lower extremity joints affected with OA, such as the knee or hip $[19,22,45-48]$. WOMAC is widely used and has been extensively validated $[49,50]$. Furthermore, according to Rogers \& Irrgang [50], the WOMAC pain and functional subscales exhibit excellent responsiveness. The minimal clinically important rehabilitation effects ranged from 0.80 to 1.01 [51].

Other quantitative functional outcomes such as walking endurance and gait speed [20,22,52-55] were measured using the 6-minute walk test [55] and Timedup-and-Go test [56].

\section{Level of physical activity}

Changes in level of PA were measured using the Seven Day Physical Activity Recall (7-day PAR) [57-59]. The 7-day PAR is a validated instrument in a calendar format in which a participant can indicate the duration (minutes per day), frequency (days per week) and intensity (multiples of basal metabolic rate). An adapted version of the 7-day PAR was included in the logbooks where PA was split into distinct walking and other physical activities.

\section{Analysis}

A repeated measure mixed model was used to assess the change in health-related QOL measures (adjusted 
AIMS2 for age and co-morbidity and SF-36) as well as clinical outcomes from baseline to 12 months (end of intervention) and to 18 months (6 month follow-up post-intervention) among three study groups. The model included variables such as intervention group, study month, and an interaction term between the intervention group and study month. Missing data was assumed to be missing at random (MAR) in order to include incomplete data. Pair wise differences comparing each group to one another (W vs. C, W vs. WB, WB vs. C) were assessed. The repeated measure mixed model was used to compare the change of outcomes from baseline to the 18-month follow-up. Statistical analyses were performed using SAS (version 9.2, SAS Institute Inc., Cary, North Carolina), and statistical significance was defined as $\mathrm{p}<0.05$.

\section{Results}

Clinical outcome measures

Quality of Life

Short-Form 36 There were no statistically significant results between the three groups for QOL measured with the SF-36 (Table 1) with the exceptions of the variables "physical role functioning", "physical role", "pain index" and "standardized physical component". At 12months, results favoured the W group for the "physical role" variable when compared to the WB group ( $\mathrm{p}=$ 0.04). At 18-months, results favoured the $W$ group for the "physical functioning" $(p=0.02)$ and "pain index" $(\mathrm{p}=0.00)$ variables when compared to the WB group. The self-directed group was also favoured when compared either to the W or WB groups for the "physical functioning" ( $\mathrm{p}=0.01)$, "pain index" $(\mathrm{p}=0.03)$ and "standardized physical component" $(\mathrm{p}=0.00)$ variables.

The selected results for SF-36 analysis were presented at baseline, at end of intervention (12 months), and at 6months post intervention (18- month follow-up). The mean values of the 8 domains of the SF-36 for the three study groups are detailed in Table 1 . After the 12-month intervention phase of the walking program, the selfdirected group $(C)$ had the highest mean ( \pm SE) "physical role" score $65.85( \pm 42.48)$. The WB group demonstrated the lowest mean pain index score 63.80 $( \pm 20.12)$. At 18 -months, the self-directed group (C) demonstrated the highest mean scores for "physical functioning" $75.69( \pm 19.65)$, "pain index" 67.44( \pm 18.32$)$, and "standardized physical component" 45.15( \pm 8.93$)$. The WB group had the lowest mean scores for "physical functioning" 63.25( \pm 25.71$)$, "pain index" 61.17( \pm 18.32$)$, and "standardized physical component" 40.91( \pm 11.04$)$.

Arthritis Impact Measurement Scale 2 (AIMS2) The AIMS2 analyses were performed at baseline, at end of intervention (12- months), and at 6-months post intervention (18- month follow-up) (Table 2). At 12months, statistical significance was observed when the WB group was compared to the self-directed group (C), mean results $( \pm$ SE) favoured the self-directed group $(C)$ for the "hand and finger" $(p=0.04)$, "social activity" $(\mathrm{p}=0.01)$, "arthritis pain" $(\mathrm{p}=0.02)$, "symptoms component" ( $\mathrm{p}=0.02)$ and "social interaction component" $(\mathrm{p}=0.01)$ variables. At 12 -months, statistical significance was only observed when the $\mathrm{W}$ group was compared to the self-directed group $(C)$, mean results $( \pm S E)$ favoured the self-directed group $(\mathrm{C})$ for the "social activity" $(\mathrm{p}=0.01)$ variable.

At 18- months, when the WB group was compared to the self-directed group $(C)$, mean results $( \pm S E)$ favoured the self-directed group for the "walking and bending" $(\mathrm{p}=0.044)$, "household tasks" $(\mathrm{p}=0.02)$, "arthritis pain" ( $p=0.03)$, "physical component" $(p=0.01)$, and "symptoms component" $(\mathrm{p}=0.03)$ variables. When the W group was compared to the WB group, results favoured the W group for the "arthritis pain" $(\mathrm{p}=0.01)$, "arthritis impact" $(p=0.01)$, "physical component" $(p=0.04)$, and "symptoms component" ( $\mathrm{p}=0.01)$ variables.

At 12-months, the WB group demonstrated the highest mean scores for the "arthritis pain" 3.79 $( \pm 2.29)$ and "symptoms component" $3.79( \pm 2.29)$ variables, and the lowest score $0.46( \pm 0.69)$ for the "hand and finger" variable. The W group had the highest mean scores for the "social activity" 4.73 $( \pm 1.57)$ and "social interaction component" 3.36 $( \pm 1.51)$ variables. The self-directed group (C) had the lowest "symptoms component" score 3.94 $( \pm 2.38)$. At 18 -months, the WB group demonstrated the highest mean scores for the "walking and bending" 3.70 $( \pm 2.40)$, "household tasks" $0.57( \pm 1.38)$, "arthritis pain" 3.64 $( \pm 2.16)$, "physical component" $1.06( \pm 0.75)$, and "symptoms component" $3.64( \pm 2.16)$ variables. The self-directed $\operatorname{group}(\mathrm{C})$ had the lowest scores for the "walking and bending" 2.708( \pm 2.11$)$, "arthritis pain" $3.40( \pm 2.23)$, "physical component" $0.68( \pm 0.61)$, and "symptoms component" $3.40( \pm 2.23)$ variables.

\section{Clinical outcomes \\ Functional status}

At 12 and 18- months, improvements were observed in each three comparative groups as the overall item scores decreased when compared to baseline (Table 3). No statistical significance was demonstrated for total WOMAC scores at 12-months. At 18 months, the self-directed (C) group demonstrated the lowest mean $( \pm \mathrm{SD})$ "pain" scores $23.50( \pm 17.78)$. The only statistically significant difference in total functional scores found between the 3 groups was at 18-months which favoured the $\mathrm{W}$ group when compared to the WB group $( \pm 16.74)$. 
Table 1 Summary of SF-36 for three study arms

\begin{tabular}{|c|c|c|c|c|c|c|c|c|c|}
\hline \multirow[t]{5}{*}{ Variables } & \multicolumn{3}{|c|}{ Baseline } & \multicolumn{3}{|c|}{12 Months } & \multicolumn{3}{|c|}{18 Months } \\
\hline & w & WB & $\mathrm{C}$ & w & WB & $\mathrm{C}$ & w & WB & $\mathrm{C}$ \\
\hline & $\mathbf{N}$ & $\mathbf{N}$ & $\mathrm{N}$ & $\mathrm{N}$ & $\mathrm{N}$ & $\mathrm{N}$ & $\mathrm{N}$ & $\mathrm{N}$ & $\mathbf{N}$ \\
\hline & Mean \pm SD & Mean \pm SD & Mean \pm SD & Mean \pm SD & Mean \pm SD & Mean \pm SD & Mean \pm Sd & Mean \pm SD & Mean \pm SD \\
\hline & & & & W Vs. C(P) & WB Vs. C(P) & WB Vs. W(P) & W Vs. C(P) & WB Vs. C(P) & WB Vs. W(P) \\
\hline \multirow[t]{3}{*}{ Physical Functioning } & 79 & 69 & 74 & 44 & 43 & 41 & 44 & 42 & 36 \\
\hline & $63.003 \pm 18.332$ & $66.449 \pm 19.157$ & $67.275 \pm 20.446$ & $70.088 \pm 18.819$ & $68.127 \pm 19.694$ & $68.171 \pm 26.381$ & $68.157 \pm 21.308$ & $63.254 \pm 25.705$ & $75.694 \pm 19.645$ \\
\hline & & & & $(0.250)$ & $(0.982)$ & $(0.234)$ & $(0.836)$ & ${ }^{*}(0.012)$ & $*(0.015)$ \\
\hline \multirow[t]{3}{*}{ Role Physical } & 78 & 69 & 74 & 44 & 44 & 41 & 44 & 42 & 36 \\
\hline & $52.350 \pm 40.064$ & $69.203 \pm 35.393$ & $56.757 \pm 39.755$ & $61.742 \pm 39.755$ & $59.659 \pm 41.138$ & $65.854 \pm 42.484$ & $57.386 \pm 40.556$ & $60.119 \pm 40.971$ & $68.519 \pm 39.345$ \\
\hline & & & & $(0.909)$ & $(0.063)$ & $*(0.044)$ & $(0.864)$ & $(0.058)$ & $(0.071)$ \\
\hline \multirow[t]{3}{*}{ Pain Index } & 78 & 69 & 74 & 44 & 44 & 41 & 44 & 42 & 36 \\
\hline & $60.487 \pm 16.040$ & $67.348 \pm 18.261$ & $62.149 \pm 18.762$ & $63.818 \pm 19.134$ & $63.795 \pm 20.124$ & $67.805 \pm 18.378$ & $65.045 \pm 18.881$ & $61.167 \pm 18.322$ & $67.444 \pm 18.315$ \\
\hline & & & & $(0.581)$ & $*(0.026)$ & $(0.082)$ & $(0.491)$ & $*(0.030)$ & $*(0.003)$ \\
\hline \multirow[t]{3}{*}{ General Health Perceptions } & 79 & 68 & 74 & 44 & 44 & 41 & 44 & 42 & 36 \\
\hline & $67.873 \pm 18.214$ & $70.838 \pm 20.465$ & $70.635 \pm 17.434$ & $67.619 \pm 17.484$ & $68.926 \pm 19.008$ & $72.000 \pm 18.358$ & $69.307 \pm 19.189$ & $66.143 \pm 20.483$ & $69.917 \pm 18.243$ \\
\hline & & & & $(0.223)$ & $(0.089)$ & $(0.605)$ & $(0.226)$ & $(0.496)$ & $(0.051)$ \\
\hline \multirow[t]{3}{*}{ Vitality } & 79 & 68 & 74 & 44 & 44 & 41 & 44 & 42 & 36 \\
\hline & $58.481 \pm 17.711$ & $60.588 \pm 21.152$ & $60.473 \pm 18.984$ & $62.045 \pm 19.628$ & $60.341 \pm 19.778$ & $64.756 \pm 18.267$ & $60.152 \pm 21.858$ & $58.929 \pm 18.791$ & $66.157 \pm 17.839$ \\
\hline & & & & $(0.856)$ & $(0.475)$ & $(0.359)$ & $(0.845)$ & $(0.517)$ & $(0.380)$ \\
\hline \multirow[t]{3}{*}{ Social Functioning } & 79 & 69 & 74 & 44 & 44 & 41 & 44 & 42 & 36 \\
\hline & $80.380 \pm 22.080$ & $85.507 \pm 18.394$ & $82.432 \pm 20.474$ & $79.545 \pm 22.889$ & $84.375 \pm 18.309$ & $85.671 \pm 20.264$ & $78.125 \pm 26.704$ & $84.226 \pm 19.533$ & $79.167 \pm 21.547$ \\
\hline & & & & $(0.266)$ & $(0.168)$ & $(0.775)$ & $(0.232)$ & $(0.735)$ & $(0.379)$ \\
\hline \multirow[t]{3}{*}{ Role Emotional } & 78 & 69 & 74 & 44 & 44 & 41 & 44 & 42 & 36 \\
\hline & $75.214 \pm 37.000$ & $84.058 \pm 29.488$ & $74.324 \pm 37.643$ & $85.606 \pm 30.835$ & $81.061 \pm 32.468$ & $82.927 \pm 35.057$ & $75.000 \pm 40.106$ & $80.159 \pm 31.286$ & $82.407 \pm 33.320$ \\
\hline & & & & $(0.949)$ & $(0.170)$ & $(0.144)$ & $(0.597)$ & $(0.290)$ & $(0.574)$ \\
\hline \multirow[t]{3}{*}{ Mental Health Index } & 78 & 68 & 74 & 44 & 44 & 41 & 44 & 42 & 36 \\
\hline & $76.500 \pm 17.558$ & $77.588 \pm 15.670$ & $80.365 \pm 13.974$ & $78.364 \pm 16.007$ & $80.000 \pm 14.823$ & $81.366 \pm 14.411$ & $77.114 \pm 17.926$ & $77.405 \pm 15.759$ & $79.333 \pm 14.890$ \\
\hline & & & & $(0.735)$ & $(0.909)$ & $(0.822)$ & $(0.597)$ & $(0.880)$ & $(0.486)$ \\
\hline \multirow[t]{3}{*}{ Health Transition Item } & 79 & 69 & 74 & 43 & 43 & 41 & 44 & 42 & $362.639 \pm 0.899$ \\
\hline & $2.975 \pm 0.987$ & $3.014 \pm 0.737$ & $2.959 \pm 0.818$ & $2.419 \pm 0.852$ & $2.698 \pm 0.914$ & $2.463 \pm 0.840$ & $2.727 \pm 0.924$ & $3.024 \pm 1.024$ & $(0.459)$ \\
\hline & & & & $(0.821)$ & $(0.564)$ & $(0.415)$ & $(0.832)$ & $(0.356)$ & \\
\hline
\end{tabular}


Table 1 Summary of SF-36 for three study arms (Continued)

Standardized Physical Component $77 \quad 68 \quad 74$

$40.516 \pm 8.598$

$41.996 \pm 9.100$

44

43

41

$42.508 \pm 9.229 \quad 42.192 \pm 10.066$

44

42

36

$\begin{array}{lll}(0.804) & (0.135) & (0.074)\end{array}$

$40.909 \pm 11.038 \quad 45.149 \pm 8.930$

(0.703) * $(0.009) \quad *(0.002)$

Standardized Mental Component 77

$68 \quad 74$

43

41

42

36

$53.819 \pm 9.852 \quad 54.476 \pm 7.329$

$55.162 \pm 8.540$

$51.993 \pm 11.000$

36

$52.914 \pm 10.845 \quad 53.812 \pm 8.639 \quad 53.556 \pm 8.995$

(0.595)

(0.436)

(0.795)

(0.688)

(0.638)

(0.937) 
Table 2 Summary of AIMS2 for three study arms

\begin{tabular}{|c|c|c|c|c|c|c|c|c|c|}
\hline \multirow[t]{5}{*}{ Variables } & \multicolumn{3}{|c|}{ Baseline } & \multicolumn{3}{|c|}{12 Months } & \multicolumn{3}{|c|}{18 Months } \\
\hline & \multirow{2}{*}{$\frac{\mathrm{W}}{\mathrm{N}}$} & \multirow{2}{*}{$\frac{\mathrm{WB}}{\mathrm{N}}$} & \multirow{2}{*}{$\frac{\mathrm{C}}{\mathrm{N}}$} & \multirow{2}{*}{$\frac{\mathrm{W}}{\mathrm{N}}$} & \multirow{2}{*}{$\frac{W B}{N}$} & \multirow{2}{*}{$\frac{\mathrm{C}}{\mathrm{N}}$} & $\mathrm{w}$ & WB & $\mathrm{C}$ \\
\hline & & & & & & & $\mathbf{N}$ & $\mathbf{N}$ & $\mathbf{N}$ \\
\hline & Mean \pm SD & Mean \pm SD & Mean \pm SD & Mean \pm SD & Mean \pm SD & Mean \pm SD & Mean \pm Sd & Mean \pm SD & Mean \pm SD \\
\hline & & & & W Vs. C(P) & $\overline{\text { WB Vs. } C(P)}$ & $\overline{\text { WB Vs. W(P) }}$ & W Vs. C(P) & $\overline{\text { WB Vs. } C(P)}$ & WB Vs. W(P) \\
\hline \multirow[t]{3}{*}{ Mobility } & 79 & 69 & 74 & 44 & 44 & 41 & 44 & 42 & 36 \\
\hline & $1.281 \pm 1.511$ & $1.058 \pm 1.621$ & $1.169 \pm 1.479$ & $0.614 \pm 1.017$ & $0.773 \pm 0.866$ & $0.720 \pm 1.090$ & $0.815 \pm 1.189$ & $0.869 \pm 1.215$ & $0.486 \pm 0.898$ \\
\hline & & & & $(0.662)$ & $(0.276)$ & $(0.121)$ & $(0.714)$ & $(0.086)$ & $(0.156)$ \\
\hline \multirow{3}{*}{$\begin{array}{l}\text { Walking } \\
\text { and Bending }\end{array}$} & 79 & 69 & 74 & 44 & 44 & 41 & 44 & 42 & 36 \\
\hline & $4.044 \pm 2.047$ & $3.513 \pm 2.538$ & $3.792 \pm 2.494$ & $3.364 \pm 2.216$ & $3.695 \pm 2.248$ & $3.085 \pm 2.490$ & $3.670 \pm 2.323$ & $3.702 \pm 2.402$ & $2.708 \pm 2.112$ \\
\hline & & & & $(0.900)$ & $(0.081)$ & $(0.094)$ & $(0.656)$ & $*(0.044)$ & (0.099) \\
\hline \multirow{3}{*}{$\begin{array}{l}\text { Hand and } \\
\text { Finger }\end{array}$} & 79 & 68 & 74 & 44 & 44 & 41 & 44 & 42 & 36 \\
\hline & $0.704 \pm 1.061$ & $0.647 \pm 1.033$ & $0.412 \pm 0.904$ & $0.602 \pm 0.931$ & $0.455 \pm 0.689$ & $0.744 \pm 1.937$ & $0.616 \pm 1.195$ & $0.693 \pm 0.917$ & $0.569 \pm 1.196$ \\
\hline & & & & $(0.127)$ & *(0.039) & $(0.573)$ & $(0.314)$ & $(0.552)$ & $(0.678)$ \\
\hline \multirow[t]{3}{*}{ Arm Function } & 79 & 68 & 74 & 44 & 44 & 41 & 43 & 42 & 36 \\
\hline & $0.363 \pm 0.795$ & $0.368 \pm 1.010$ & $0.264 \pm 0.642$ & $0.295 \pm 0.726$ & $0.591 \pm 1.448$ & $0.220 \pm 0.699$ & $0.581 \pm 1.170$ & $0.405 \pm 0.843$ & $0.194 \pm 0.511$ \\
\hline & & & & $(0.451)$ & $(0.253)$ & $(0.673)$ & $(0.175)$ & $(0.635)$ & $(0.369)$ \\
\hline \multirow[t]{3}{*}{ Self Care } & 79 & 69 & 74 & 44 & 44 & 41 & 43 & 42 & 36 \\
\hline & $0.391 \pm 1.632$ & $0.045 \pm 0.247$ & $0.194 \pm 1.179$ & $0.114 \pm 0.509$ & $0.028 \pm 0.132$ & $0.091 \pm 0.358$ & $0.087 \pm 0.572$ & $0.104 \pm 0.435$ & $0.000 \pm 0.000$ \\
\hline & & & & $(0.801)$ & $(0.726)$ & $(0.541)$ & $(0.541)$ & $(0.409)$ & $(0.147)$ \\
\hline \multirow{3}{*}{$\begin{array}{l}\text { Household } \\
\text { Tasks }\end{array}$} & 79 & 69 & 74 & 44 & 44 & 41 & 44 & 42 & 36 \\
\hline & $0.539 \pm 1.093$ & $0.272 \pm 0.806$ & $0.450 \pm 1.549$ & $0.341 \pm 1.004$ & $0.114 \pm 0.279$ & $0.274 \pm 0.713$ & $0.412 \pm 0.933$ & $0.565 \pm 1.379$ & $0.122 \pm 0.468$ \\
\hline & & & & $(0.924)$ & $(0.879)$ & $(0.802)$ & $(0.679)$ & $*(0.024)$ & $(0.053)$ \\
\hline \multirow[t]{3}{*}{ Social Activity } & 79 & 69 & 74 & 44 & 43 & 41 & 44 & 42 & 36 \\
\hline & $4.530 \pm 1.723$ & $4.449 \pm 1.774$ & $4.639 \pm 2.050$ & $4.727 \pm 1.573$ & $4.453 \pm 1.886$ & $3.732 \pm 2.188$ & $4.625 \pm 1.840$ & $4.720 \pm 1.860$ & $4.281 \pm 2.141$ \\
\hline & & & & $*(0.013)$ & $*(0.006)$ & $(0.767)$ & $(0.944)$ & $(0.233)$ & $(0.187)$ \\
\hline \multirow{3}{*}{$\begin{array}{l}\text { Support } \\
\text { From Family }\end{array}$} & 79 & 69 & 74 & 44 & 43 & 40 & 44 & 42 & 36 \\
\hline & $1.907 \pm 2.286$ & $1.778 \pm 1.938$ & $2.204 \pm 2.158$ & $1.989 \pm 2.269$ & $2.253 \pm 2.379$ & $2.328 \pm 2.356$ & $1.932 \pm 2.437$ & $1.979 \pm 2.052$ & $2.743 \pm 2.606$ \\
\hline & & & & $(0.605)$ & $(0.100)$ & $(0.237)$ & $(0.308)$ & $(0.824)$ & $(0.414)$ \\
\hline \multirow[t]{3}{*}{ Arthritis Pain } & 79 & 69 & 73 & 44 & 44 & 41 & 443. & 42 & 35 \\
\hline & $4.396 \pm 1.907$ & $3.813 \pm 2.175$ & $4.270 \pm 2.426$ & $3.494 \pm 2.383$ & $3.793 \pm 2.285$ & $3.494 \pm 2.383$ & $440 \pm 2.409$ & $3.642 \pm 2.157$ & $3.400 \pm 2.228$ \\
\hline & & & & $(0.523)$ & ${ }^{*}(0.019)$ & $(0.075)$ & $(0.836)$ & $*(0.030)$ & $*(0.013)$ \\
\hline Work & 37 & 25 & 40 & 15 & 11 & 18 & 12 & 9 & 14 \\
\hline & $2.095 \pm 2.302$ & $1.975 \pm 2.193$ & $1.828 \pm 1.958$ & $1.542 \pm 1.828$ & $0.625 \pm 0.791$ & $1.875 \pm 1.561$ & $2.188 \pm 2.450$ & $2.083 \pm 1.849$ & $1.830 \pm 1.826$ \\
\hline & & & & $(0.536)$ & $(0.171)$ & $(0.428)$ & $(0.730)$ & $(0.825)$ & $(0.608)$ \\
\hline Level of Tension & 79 & 69 & 74 & 44 & 43 & 40 & 44 & 42 & 36 \\
\hline & $3.327 \pm 1.850$ & $3.338 \pm 1.900$ & $3.030 \pm 1.718$ & $3.085 \pm 1.692$ & $3.078 \pm 1.731$ & $2.972 \pm 1.897$ & $3.313 \pm 1.969$ & $3.012 \pm 1.799$ & $2.815 \pm 1.601$ \\
\hline & & & & $(0.728)$ & $(0.810)$ & $(0.916)$ & $(0.804)$ & $(0.825)$ & $(0.628)$ \\
\hline Mood & 79 & 69 & 74 & 44 & 42 & 40 & 44 & 42 & 36 \\
\hline & $2.036 \pm 1.548$ & $1.937 \pm 1.479$ & $1.726 \pm 1.168$ & $1.795 \pm 1.636$ & $1.818 \pm 1.324$ & $1.478 \pm 1.278$ & $1.795 \pm 1.909$ & $1.893 \pm 1.290$ & $1.531 \pm 1.301$ \\
\hline & & & & $(0.686)$ & $(0.224)$ & $(0.397)$ & $(0.622)$ & $(0.449)$ & $(0.194)$ \\
\hline Satisfaction & 79 & 69 & 74 & 43 & 44 & 41 & 44 & 42 & 36 \\
\hline & $2.567 \pm 1.580$ & $2.746 \pm 1.762$ & $2.523 \pm 1.458$ & $2.113 \pm 1.818$ & $2.333 \pm 1.728$ & $2.127 \pm 1.640$ & $2.183 \pm 2.080$ & $2.657 \pm 1.885$ & $1.934 \pm 1.570$ \\
\hline & & & & $(0.529)$ & $(0.800)$ & $(0.704)$ & $(0.599)$ & $(0.305)$ & $(0.108)$ \\
\hline
\end{tabular}


Table 2 Summary of AIMS2 for three study arms (Continued)

\begin{tabular}{|c|c|c|c|c|c|c|c|c|c|}
\hline \multirow{3}{*}{$\begin{array}{l}\text { Health } \\
\text { Perception }\end{array}$} & 79 & 68 & 72 & 44 & 43 & 39 & 42 & 42 & 34 \\
\hline & $3.890 \pm 2.417$ & $3.340 \pm 2.159$ & $3.572 \pm 2.464$ & $3.644 \pm 2.369$ & $3.495 \pm 1.778$ & $3.340 \pm 1.877$ & $3.340 \pm 1.952$ & $3.658 \pm 2.310$ & $3.438 \pm 1.926$ \\
\hline & & & & $(0.420)$ & $(0.117)$ & $(0.418)$ & $(0.311)$ & $(0.473)$ & $(0.073)$ \\
\hline \multirow[t]{3}{*}{ Arthritis Impact } & 76 & 65 & 70 & 39 & 40 & 36 & 34 & 39 & 30 \\
\hline & $3.191 \pm 2.757$ & $2.769 \pm 2.259$ & $2.929 \pm 2.407$ & $2.372 \pm 1.809$ & $2.500 \pm 2.334$ & $2.222 \pm 1.962$ & $1.985 \pm 2.112$ & $3.013 \pm 2.512$ & $2.250 \pm 1.897$ \\
\hline & & & & $(0.431)$ & $(0.077)$ & $(0.308)$ & $(0.317)$ & $(0.140)$ & ${ }^{*}(0.011)$ \\
\hline \multirow{3}{*}{$\begin{array}{l}\text { Physical } \\
\text { Component }\end{array}$} & 79 & 68 & 74 & 44 & 44 & 41 & 43 & 42 & 36 \\
\hline & $1.224 \pm 0.830$ & $0.991 \pm 0.777$ & $1.047 \pm 0.747$ & $0.883 \pm 0.845$ & $0.943 \pm 0.549$ & $0.856 \pm 0.771$ & $1.039 \pm 1.005$ & $1.057 \pm 0.753$ & $0.676 \pm 0.612$ \\
\hline & & & & $(0.554)$ & $(0.352)$ & $(0.121)$ & $(0.562)$ & $*(0.011)$ & $*(0.040)$ \\
\hline \multirow{3}{*}{$\begin{array}{l}\text { Affect } \\
\text { Component }\end{array}$} & 79 & 69 & 74 & 44 & 42 & 40 & 44 & 42 & 36 \\
\hline & $2.681 \pm 1.570$ & $2.637 \pm 1.505$ & $2.378 \pm 1.344$ & $2.440 \pm 1.502$ & $2.438 \pm 1.436$ & $2.225 \pm 1.445$ & $2.554 \pm 1.806$ & $2.452 \pm 1.423$ & $2.173 \pm 1.368$ \\
\hline & & & & $(0.937)$ & $(0.690)$ & $(0.623)$ & $(0.934)$ & $(0.835)$ & $(0.762)$ \\
\hline \multirow{3}{*}{$\begin{array}{l}\text { Symptoms } \\
\text { Component }\end{array}$} & 79 & 69 & 73 & 44 & 44 & 41 & 44 & 42 & 35 \\
\hline & $4.372 \pm 1.907$ & $3.813 \pm 2.175$ & $4.270 \pm 2.426$ & $3.523 \pm 2.360$ & $3.793 \pm 2.285$ & $3.494 \pm 2.383$ & $3.440 \pm 2.409$ & $3.642 \pm 2.157$ & $3.400 \pm 2.228$ \\
\hline & & & & $(0.523)$ & ${ }^{*}(0.019)$ & $(0.075)$ & $(0.836)$ & $*(0.030)$ & $*(0.013)$ \\
\hline \multirow{3}{*}{$\begin{array}{l}\text { Social Interaction } \\
\text { Component }\end{array}$} & 79 & 69 & 74 & 44 & 43 & 40 & 44 & 42 & 36 \\
\hline & $3.241 \pm 1.578$ & $3.114 \pm 1.552$ & $3.421 \pm 1.614$ & $3.358 \pm 1.506$ & $3.353 \pm 1.698$ & $3.033 \pm 1.977$ & $3.278 \pm 1.906$ & $3.350 \pm 1.556$ & $3.512 \pm 1.965$ \\
\hline & & & & $(0.081)$ & $*(0.006)$ & $(0.268)$ & $(0.388)$ & $(0.539)$ & $(0.127)$ \\
\hline \multirow[t]{3}{*}{ Role Component } & 37 & 25 & 40 & 15 & 11 & 18 & 12 & 9 & 14 \\
\hline & $2.095 \pm 2.302$ & $1.975 \pm 2.193$ & $1.828 \pm 1.958$ & $1.542 \pm 1.828$ & $0.625 \pm 0.791$ & $1.875 \pm 1.561$ & $2.188 \pm 2.450$ & $2.083 \pm 1.849$ & $1.830 \pm 1.826$ \\
\hline & & & & $(0.536)$ & $(0.171)$ & $(0.428)$ & $(0.730)$ & $(0.825)$ & (0.608) \\
\hline
\end{tabular}

Table 3 Summary of WOMAC for three study arms

\begin{tabular}{|c|c|c|c|c|c|c|c|c|c|}
\hline \multirow[t]{5}{*}{ Variables } & \multicolumn{3}{|c|}{ Baseline } & \multicolumn{3}{|c|}{12 Months } & \multicolumn{3}{|c|}{18 Months } \\
\hline & w & WB & $\mathrm{C}$ & w & WB & $\mathrm{C}$ & w & WB & C \\
\hline & $\mathrm{N}$ & $\mathrm{N}$ & $\mathrm{N}$ & $\mathrm{N}$ & $\mathrm{N}$ & $\mathrm{N}$ & $\mathrm{N}$ & $\mathrm{N}$ & $\mathrm{N}$ \\
\hline & Mean \pm SD & Mean \pm SD & Mean \pm SD & Mean \pm SD & Mean \pm SD & Mean \pm SD & Mean \pm SD & Mean \pm SD & Mean \pm SD \\
\hline & & & & W Vs. C(P) & WB Vs. C(P) & WB Vs. W(P) & W Vs. C(P) & WB Vs. C(P) & WB Vs. W(P) \\
\hline \multirow[t]{3}{*}{ Pain } & 79 & 69 & 73 & 43 & 42 & 41 & 43 & 42 & 35 \\
\hline & $31.15 \pm 14.29$ & $26.81 \pm 14.92$ & $30.30 \pm 16.47$ & $24.65 \pm 15.78$ & $25.32 \pm 15.98$ & $25.00 \pm 19.44$ & $23.60 \pm 15.09$ & $26.16 \pm 17.97$ & $23.50 \pm 17.78$ \\
\hline & & & & $(0.572)$ & $(0.238)$ & $(0.522)$ & $(0.863)$ & $(0.157)$ & $(0.096)$ \\
\hline \multirow[t]{3}{*}{ Stiffness } & 79 & 69 & 71 & 44 & 41 & 40 & 43 & 42 & 35 \\
\hline & $38.90 \pm 18.01$ & $36.41 \pm 18.90$ & $39.08 \pm 19.81$ & $30.96 \pm 22.31$ & $30.79 \pm 19.58$ & $28.43 \pm 20.41$ & $29.94 \pm 20.43$ & $31.40 \pm 20.75$ & $27.14 \pm 18.80$ \\
\hline & & & & $(0.125)$ & $(0.405)$ & $(0.486)$ & $(0.494)$ & $(0.423)$ & $(0.890)$ \\
\hline \multirow{3}{*}{$\begin{array}{l}\text { Physical } \\
\text { Function }\end{array}$} & 79 & 68 & 72 & 44 & 38 & 40 & 43 & 42 & 35 \\
\hline & $28.16 \pm 15.41$ & $27.65 \pm 18.22$ & $26.89 \pm 16.34$ & $24.48 \pm 13.79$ & $25.27 \pm 15.70$ & $25.06 \pm 13.53$ & $18.20 \pm 14.63$ & $24.15 \pm 17.24$ & $19.40 \pm 17.08$ \\
\hline & & & & $(0.672)$ & $(0.903)$ & $(0.763)$ & $(0.582)$ & $(0.381)$ & $(0.140)$ \\
\hline \multirow{3}{*}{$\begin{array}{l}\text { Total } \\
\text { WOMAC Score }\end{array}$} & 79 & 68 & 70 & 43 & 41 & 40 & 43 & 42 & 35 \\
\hline & $29.70 \pm 14.09$ & $28.27 \pm 16.42$ & $28.95 \pm 15.28$ & $21.05 \pm 13.62$ & $23.60 \pm 13.61$ & $22.32 \pm 17.77$ & $20.30 \pm 13.97$ & $25.58 \pm 16.60$ & $20.90 \pm 16.74$ \\
\hline & & & & $(0.612)$ & $(0.475)$ & $(0.821)$ & $(0.464)$ & $(0.129)$ & $*(0.019)$ \\
\hline
\end{tabular}

W: Walking only group; WB: Walking and Behavioural Group; C: Self-directed group (unsupervised/self-directed); N: number of subjects in each comparative group; SD: standard deviation; vs: versus; data is presented as mean (standard deviation); P: p-value (statistical significance); * Statistically significant; WOMAC: Western Ontario MacMaster Osteoarthritis Index. 


\section{Walking endurance}

There were no statistically significant results for the 6-min walk test among the three groups after 12 and 18- months (Table 4). Distance (in meters) increased during the 6- minute walk test at both 12 and 18 months when compared to baseline among all three groups (Table 5). At 12- months, individuals in the $\mathrm{W}$ group had the highest score $524.86( \pm 106.52)$. The self-directed $(C)$ group had the highest score $540.35( \pm 103.37)$ at 18 months.

\section{Gait speed}

There were no statistically significant results at both 12 months and 18-months for gait speed (Table 5). These results were similar to walking endurance, as gait speed was calculated using the 6 minute walk test. At 12 months, the W group had the highest score $1.458( \pm 0.296)$. At 18 -months the self-directed $(\mathrm{C})$ group had the highest score $1.501( \pm 0.287)$.

\section{Timed up and Go}

There were no statistically significant results for the Timed up and Go among the three groups at 12 and 18- months (Table 6). At 12 and 18-months, Timed up and Go scores (time in seconds) decreased in all three groups when compared to baseline (Table 4). Timed up and Go scores were lowest for all 3 groups at 12 months when compared to 18 months. At 12 months, the WB group had the lowest score $8.10( \pm 1.54)$ compared to the other two groups. At 18 months, the self-directed (C) group had the lowest score $7.88( \pm 1.89)$.

\section{Level of physical activity}

After participating in the 12-month walking program [3-5], the WB group demonstrated the highest increase in activity level (Table 7). Statistical significance was not reached for "Leisure-Time Physical Activity" variables. At 12 months, the WB demonstrated the highest mean scores for "Leisure-Time Physical Activity Walking only" $13.89( \pm 12.40)$ and for "Leisure-Time Physical Activity Walking and other activities" 19.77 ( \pm 15.85$)$. At 18 months, the self-directed $(C)$ group demonstrated the highest mean score of $16.88( \pm 17.50)$ for "Leisure-Time Physical Activity Walking only" and $24.18( \pm 25.59)$ for "Leisure-Time Physical Activity Walking \& other activities". At 12 and 18 months, the WB group had the highest mean score for "Occupational/Domestic Activity Walking only" $33.00( \pm 70.75)$ and $33.07( \pm 39.04)$ and the highest "Occupational/Domestic Activity Walking and other activities" mean score $41.48( \pm 61.94)$. Statistical significance was demonstrated for "Occupational/ Domestic Activity Walking and other activities" mean scores at 12 - months $27.99( \pm 37.28)$ and 18 months $26.13( \pm 15.64)$ for the WB group.

\section{Discussion}

We attempted to find the best way to implement a proven effective SCAWP from a clinical point of view [3-5]. As expected from the Ottawa Panel CPG on SCAWP [3-5], QOL as well as clinical outcomes of this RCT improved among participants with mild to moderate $\mathrm{OA}$ in each of the three comparative groups. However, very few statistically significant differences were observed for QOL and clinical outcomes over a long duration after the 12-month intervention phase and the 18-month follow-up phase. Results varied among the three groups depending on which outcomes were considered.

Participants from each group showed improvements in QOL and clinical outcomes measurements as they benefited from the walking component which has found be effective according to the Ottawa Panel CPGs [3-5]. The behavioural component facilitated the implementation of The Ottawa Panel CPG on SCAWP [3-5] over a shortterm period (See part I), but did not have a direct impact on QOL and clinical outcomes [6].

\section{Quality of life (QOL) outcomes}

Various PA programs, including walking programs for OA, have improved QOL when compared to a control group with no walking involvement over a short-term period [18-24]. However, this effect was not maintained after a period of unsupervised PA at 6 months [24], 9 months [20], and 18 months [25]. This RCT used a selfdirected walking group as comparator.

Surprisingly, all statistically significant results favoured the self-directed group $(C)$ group compared to the two walking groups (W and WB groups) with QOL improvements at 12 and 18 months. The behavioural component of the WB group was expected to improve participant adherence to the program over a long period of time, allowing participants to benefit from the proven effective walking program by improving and maintaining QOL and clinical outcomes. Some explanations as to why participants in the self-directed $(C)$ group demonstrated a higher QOL score may be due to the development of fewer health problems and fewer difficulties with transportation resulting in less attrition to the study. In addition, participants in the self-directed (C) group may not have experienced as many barriers as the other two groups in order to engage in walking activities for at least 3 times a week. This group had the comfort of being around their own environment, especially for those who were not retired and still working. These facilitators may have contributed to the improvement of the participants' QOL compared to the other groups which were required to travel regularly to the walking club. 
Table 4 Summary of 6-minute walking for three study arms (6 Minute Walk Test)

\begin{tabular}{|c|c|c|c|c|c|c|c|c|c|}
\hline & \multicolumn{3}{|c|}{ Baseline } & \multicolumn{3}{|c|}{12 Months } & \multicolumn{3}{|c|}{18 Months } \\
\hline & w & WB & C & w & WB & $C$ & w & WB & $\mathrm{C}$ \\
\hline & $\mathrm{N}$ & $\mathrm{N}$ & $\mathrm{N}$ & $\mathrm{N}$ & $\mathrm{N}$ & $\mathrm{N}$ & $\mathrm{N}$ & $\mathbf{N}$ & $\mathrm{N}$ \\
\hline & Mean \pm SD & Mean \pm SD & Mean \pm SD & Mean \pm SD & Mean \pm SD & Mean \pm SD & Mean \pm SD & Mean \pm SD & Mean \pm SD \\
\hline & & & & W Vs. C(P) & WB Vs. C(P) & WB Vs. W(P) & W Vs. C(P) & WB Vs. C(P) & WB Vs. W(P) \\
\hline \multirow[t]{3}{*}{ 6-min walk } & 79 & 68 & 74 & 44 & 41 & 40 & 42 & 39 & 35 \\
\hline & $456.45 \pm 87.62$ & $475.27 \pm 90.77$ & $490.89 \pm 99.30$ & $524.86 \pm 106.52$ & $509.41 \pm 82.43$ & $520.52 \pm 115.14$ & $492.91 \pm 86.95$ & $500.15 \pm 77.46$ & $540.35 \pm 103.37$ \\
\hline & & & & 0.063 & 0.535 & 0.215 & 0.366 & 0.318 & 0.902 \\
\hline
\end{tabular}

W: Walking only group; WB: Walking and Behavioural Group; C: Self-directed group (unsupervised/self-directed); N: number of subjects in each comparative group; SD: standard deviation; vs: versus; data is presented as mean (standard deviation); P: p-value (statistical significance); ${ }^{*}$ Statistically significant; $6 \mathrm{mwt}$ : 6-min walk test (walking endurance). 
Table 5 Summary of Gait Speed for three study arms

\begin{tabular}{|c|c|c|c|c|c|c|c|c|c|}
\hline & \multicolumn{3}{|c|}{ Baseline } & \multicolumn{3}{|c|}{12 Months } & \multicolumn{3}{|c|}{18 Months } \\
\hline & w & WB & $\mathrm{C}$ & w & WB & $\mathrm{H}$ & w & WB & $\mathrm{C}$ \\
\hline & $\mathrm{N}$ & $\mathrm{N}$ & $\mathrm{N}$ & $\mathrm{N}$ & $\mathrm{N}$ & $\mathrm{N}$ & $\mathrm{N}$ & $\mathrm{N}$ & $\mathrm{N}$ \\
\hline & Mean \pm SD & Mean \pm SD & Mean \pm SD & Mean \pm SD & Mean \pm SD & Mean \pm SD & Mean \pm SD & Mean \pm SD & Mean \pm SD \\
\hline & & & & W Vs. C(P) & WB Vs. C(P) & WB Vs. W(P) & W Vs. C(P) & WB Vs. C(P) & WB Vs. W(P) \\
\hline Gait Speed & 79 & 68 & 74 & 44 & 41 & 40 & 42 & 39 & 35 \\
\hline & $1.268 \pm 0.243$ & $1.320 \pm 0.252$ & $1.364 \pm 0.276$ & $1.458 \pm 0.296$ & $1.415 \pm 0.229$ & $1.446 \pm 0.320$ & $1.369 \pm 0.242$ & $1.389 \pm 0.215$ & $1.501 \pm 0.287$ \\
\hline & & & & $(0.063)$ & $(0.535)$ & $(0.215)$ & $(0.366)$ & $(0.318)$ & $(0.902)$ \\
\hline
\end{tabular}

W: Walking only group; WB: Walking and Behavioural Group; C: Self-directed group (unsupervised/self-directed); N: number of subjects in each comparative group; SD: standard deviation; vs: versus; data is presented as mean (standard deviation); P: p-value (statistical significance); ${ }^{*}$ Statistically significant.

Although the behavioural component of the WB group demonstrated the highest compliance rate for the first three months (Part I), they did not exhibit the highest QOL scores [6]. The self-directed (C) group contained the highest dropout rate, leading one to wonder if only participants with a higher QOL decided to adhere to regular walking.

\section{Clinical outcomes}

The results of primary RCTs which served to develop Ottawa Panel CPGs [3-5] revealed that 10-60 minute aerobic walking programs for OA are proven effective to reduce pain intensity $[18,22,47,52,53,60-62]$, to reduce morning stiffness $[20]$, to increase strength $[55,63]$, to improve walking endurance $[52,64]$, to better manage stairs climbing $[59,60,62]$, to increase the number of steps performed per day [62], to build self-efficacy on stairs and in walking [65], to improve functional status $[24,47,59,64-66]$ and contribute to enhance QOL $[20,24,47,52,67]$ when compared to a control group with no involvement of walking.

The nature of the walking program involved in the three comparative groups of this KT RCT demonstrated no differences between groups. The results of Evick et al. [47] revealed that a home-based exercise program is as effective as a supervised facility-based walking program for significantly improving pain relief, physical function, and quality of life at follow-up 3 months. Talbot et al. [55] observed that a home-based walking combined with education compared to education only improved the number of steps walked per day, improved the amount of time to climb stairs, and improved strength after the 12 week intervention and at follow-up 3 - months post-intervention. The combined walking and education group also demonstrated improved pain relief at follow-up 3-months post intervention. Similar to a study by Minor et al. [20], the self-directed (C) group which was home-based, demonstrated the greatest improvements in clinical outcomes.

PA creates a motor evoked potential drop which decreases the corticomotor excitability which consequently reduces pain [68]. In a trial using rats, exercise has been shown to reverse signs of neuropathic pain and increase endogenous opioid content in brainstem regions which are important for pain modulation [69,70]. Several PA programs, including walking, have been shown to result in decreased pain in the lower extremities in those suffering from OA $[18,20,22,47,53,67,71,72]$. In these studies, the duration of the programs varied between 2 and 6 months. The study by Minor [20] demonstrated that the reduction in pain persisted after completion of the supervised aerobic walking program (3 and 9-month follow-up) if subjects continued to engage in PA. In this RCT, knee pain

Table 6 Summary of Timed Up and Go for three study arms

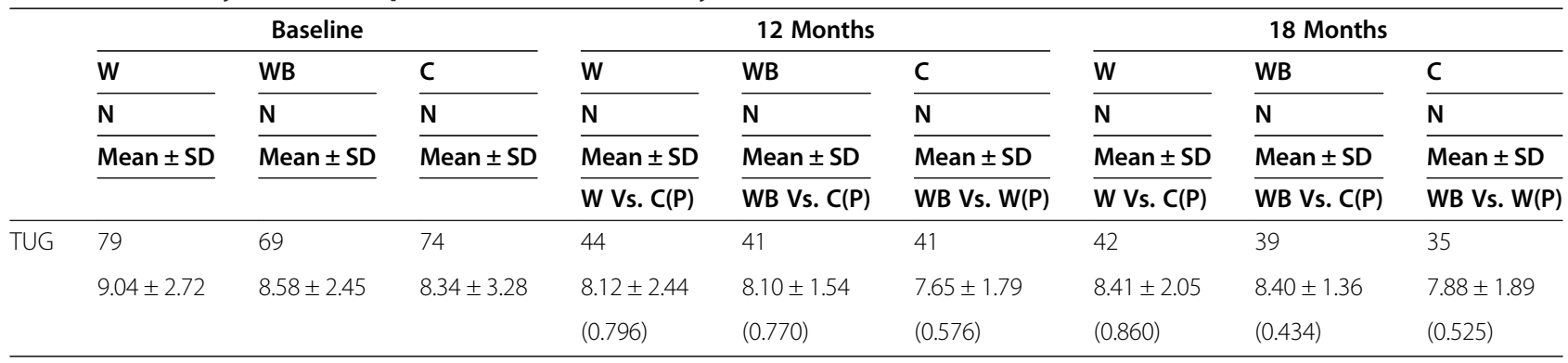


Table 7 Summary of 7 day Physical Activity Recall for three study arms

\begin{tabular}{|c|c|c|c|c|c|c|c|c|c|}
\hline \multirow[t]{5}{*}{ Variables } & \multicolumn{3}{|c|}{ Baseline } & \multicolumn{3}{|c|}{ 12Months } & \multicolumn{3}{|c|}{ 18Month } \\
\hline & w & WB & $C$ & w & WB & C & w & WB & $\mathrm{C}$ \\
\hline & $\mathrm{N}$ & $\mathrm{N}$ & $\mathrm{N}$ & $\mathrm{N}$ & $\mathrm{N}$ & $\mathrm{N}$ & $\mathrm{N}$ & $\mathrm{N}$ & $\mathrm{N}$ \\
\hline & Mean \pm SD & Mean \pm SD & Mean \pm SD & Mean \pm SD & Mean \pm SD & Mean \pm SD & Mean \pm SD & Mean \pm SD & Mean \pm SD \\
\hline & & & & W Vs. C(P) & $\overline{\text { WB Vs. C(P) }}$ & $\overline{W B}$ Vs. W(P) & W Vs. C(P) & $\overline{\text { WB Vs. } C(P)}$ & $\overline{\text { WB Vs. W(P) }}$ \\
\hline \multirow{3}{*}{$\begin{array}{l}\text { LTA Walking } \\
\text { Only }\end{array}$} & 63 & 49 & 56 & 42 & 37 & 38 & 40 & 33 & 32 \\
\hline & $12.09 \pm 13.09$ & $13.92 \pm 16.50$ & $12.45 \pm 14.96$ & $12.22 \pm 7.86$ & $13.89 \pm 12.40$ & $12.68 \pm 11.20$ & $12.20 \pm 9.90$ & $16.40 \pm 18.72$ & $16.88 \pm 17.50$ \\
\hline & & & & $(0.816)$ & $(0.816)$ & $(0.987)$ & $(0.261)$ & $(0.932)$ & $(0.303)$ \\
\hline \multirow{3}{*}{$\begin{array}{l}\text { LTA Walking } \\
+ \text { Other }\end{array}$} & 69 & 61 & 62 & 43 & 38 & 41 & 41 & 35 & 33 \\
\hline & $16.18 \pm 21.49$ & $16.43 \pm 18.86$ & $19.74 \pm 22.87$ & $15.34 \pm 10.23$ & $19.77 \pm 15.85$ & $16.01 \pm 14.14$ & $16.46 \pm 13.17$ & $22.15 \pm 21.21$ & $24.18 \pm 25.59$ \\
\hline & & & & $(0.633)$ & $(0.139)$ & $(0.294)$ & $(0.404)$ & $(0.602)$ & $(0.165)$ \\
\hline \multirow{3}{*}{$\begin{array}{l}\text { ODA Walking } \\
\text { Only }\end{array}$} & 35 & 28 & 34 & 9 & 11 & 13 & 17 & 14 & 8 \\
\hline & $20.75 \pm 25.69$ & $11.92 \pm 12.42$ & $18.17 \pm 25.98$ & $17.10 \pm 21.03$ & $33.00 \pm 70.75$ & $11.05 \pm 8.39$ & $22.33 \pm 26.10$ & $33.07 \pm 39.04$ & $12.04 \pm 5.64$ \\
\hline & & & & $(0.675)$ & $*(0.040)$ & $(0.113)$ & $(0.601)$ & $(0.076)$ & $(0.140)$ \\
\hline \multirow{3}{*}{$\begin{array}{l}\text { ODA Walking } \\
\text { +Other }\end{array}$} & 60 & 47 & 55 & 26 & 24 & 30 & 29 & 24 & 19 \\
\hline & $31.31 \pm 36.00$ & $19.77 \pm 25.61$ & $26.85 \pm 30.88$ & $22.63 \pm 20.97$ & $41.48 \pm 61.94$ & $27.99 \pm 37.28$ & $23.34 \pm 22.40$ & $27.97 \pm 33.15$ & $26.13 \pm 15.64$ \\
\hline & & & & $(0.273)$ & $*(0.047)$ & $*(0.003)$ & $(0.198)$ & $(0.331)$ & $*(0.021)$ \\
\hline
\end{tabular}

W :Walking only group; WB: Walking and Behavioural Group; C: Self-directed group (unsupervised/self-directed); N: number of subjects in each comparative group; SD: standard deviation; vs: versus; data is presented as mean (standard deviation); p: p-value (statistical significance); * Statistically significant; ODA: Other domestic activities; LTA: Leisure time activities.

reduction, decreased morning stiffness and improved functional status were observed in all three comparative groups.

Surprisingly, there were no statistically significant results for "Leisure-Time Physical Activity" compared to "Occupational/Domestic Activity". One reason for these results may be that long term goal setting, included in the Pace-ex program for those in the WB group, were not based solely on walking activities and included any daily activities (e.g. weight loss). The two other comparative groups did not contain a goal setting intervention. In addition, baseline results illustrated that participants in the self-directed $(C)$ group received a higher level of education. Strong associations have been found between level of education, physical fitness level, and amount of leisure activity.

\section{Limitations}

A major limitation of this study was the high attrition rate in each implementation and dissemination groups. As a result, validity of long-term results at 12-months end of intervention and at 18-months follow-up is questionable. Participants in the self-directed (C) group had initial lower values (poorer QOL) while participants in WB group demonstrated higher baseline values (higher QOL) for the physical components of the SF-36 and AIMS2 questionnaires. This situation may have lead to a potential bottom effect, creating larger room for QOL (SF-36) improvements for the self-directed (C) group and a potential ceiling effect for the WB group, resulting in smaller incremental improvements for QOL.

The use of the AIMS2 questionnaire among this specific population is debatable. The study subjects enrolled in this RCT were quite functional according to our inclusion criteria. They had to experience a low level degree of pain intensity and must have been able to walk for more than 20 minutes consecutively. AIMS2 is a QOL measurement designed for individuals with more severe physical conditions. This may have led to a potential ceiling effect on certain sub-scales such as the physical components.

Another limitation may have been the nature of the self-directed (C) group. The intervention among this group consisted solely of the use of a pamphlet on walking. As a result, the self-directed (C) group was not an inactive group, and walked regularly according to their self-reported logbook reports. We believe that the selfdirected group (C) benefited just as much as the other two groups from the positive effects of walking.

Lastly, there were missing data from the 7-day PAR questionnaire as not all participants completed their logbooks and had difficulties remembering which activities were performed, resulting in a potential recall bias.

\section{Implications}

This RCT was a long-term KT as well as compliance study which addressed questions of clinical and scientific importance. This study was designed to improve the understanding of efficacy of KT strategies to promote 
the adoption and maintenance of a community-based proven effective walking program for $\mathrm{OA}$ in order to improve the QOL of participants. Results of this study revealed that a community-based location or homebased walking program may be an effective strategy to manage OA of the knee.

\section{Conclusions}

The three walking groups demonstrated globally equivalent results for the implementation of KT strategies to improve QOL and clinical outcomes over a long-term period (12 and 18-months).

\begin{abstract}
Abbreviations
OA: Osteoarthritis; QOL: Quality of life; RCT: Randomized controlled trial; SCAWP: Supervised community-based aerobic walking program; CPG: Clinical practice guideline; KT: Knowledge translation; KTAC: Knowledge Translation Action Cycle; EBCPG: Evidence-based clinical practice guidelines; CONSORT: Consolidated Standards of Reporting Trials; WB (group): Walking and behavioural intervention; W (group): Walking intervention; $C$ (group): Self-directed control intervention; PA: Physical activity; PACEex: Program for Arthritis Control through Education and Exercise; AIMS2: Arthritis Impact Measurement Scale 2; SF-36: Short-Form 36 Health Survey; WOMAC: Western Ontario and McMaster Universities Osteoarthritis Index; MAR: Missing at random; CIHR: Canadian Institutes of Health Research.
\end{abstract}

\section{Competing interests}

The authors declare that they have no competing interests.

\section{Authors' contributions}

Dr. LB is a Full Professor of Rehabilitation, an epidemiologist and the principal investigator of this study and primary author of this manuscript. Dr. GAW, the co-principal investigator of this study, is senior biostatistician and director, Cardiovascular Research Methods Centre at the University of Ottawa Heart Institute, and is a leading expert in the design and analysis of clinical trials. He provided assistance with the methodology and statistical analysis of the study. Dr. GPK is a Full Professor of Physiology at the University of Ottawa, and director of the university's Human Performance and Environmental Medicine Research Laboratory and Professional Fitness and Lifestyle Consultant Certification Training program. He assisted with the methodology of the study. Dr. RR is a senior researcher at the University of Ottawa Heart Institute and provided experience in applying innovative behavioural approaches aimed at increasing PA in healthy and chronic diseased populations. Dr. AM is a health economist and assisted with the economic evaluation concept in the original proposal. Dr. PT is a rheumatologist, an epidemiologist and chief of the Cochrane Musculoskeletal Group. He has experience in conducting RCTs and meta-analyses. He provided assistance with OA outcome measures in the study. Dr. MH \& Ms. CM developed the PACEex program and was in charge of training the physical activity specialist. Mr. GDA was the research coordinator of this study and assisted with the writing of this manuscript. Ms. LC is a biostatistician and performed the analyses of this study. All authors read and approved the final manuscript.

\section{Authors' information}

Lucie Brosseau (Ph.D.), School of Rehabilitation Sciences, Faculty of Health Sciences, University of Ottawa, 451 Smyth Road, Ottawa, Ontario, Canada. K1H 8M5.Telephone number: (613) 562-5800, ext. 8015; Fax number: (613) 562-5428; E-Mail: Lucie.Brosseau@uottawa.ca

\section{Acknowledgements}

The authors are indebted to the study participants and to the research personnel: Ms. S. Sinclair, Ms. A. Marshall, Ms. C. Cohoon, Ms. K. Hidalgo, Mr. S. Nagaraja, Ms. L. Loew, Ms. J. Larose, Mr. Danijel Sredic, Mr. A. Teav, Ms. G. Ménard, Mr. JF PorteLance, Ms. J. Smoljanic, Ms. Ivan, Ms. D. Bédard, Mr. M. Abdi, Mr. B. McCullough, Mr. E.Gannon, Mr. R.Briones, The authors are also thankful indebted to Dr. R. Sigal (M.D., M.Sc.), Dr. C. Blanchard (Ph.D.), Dr. J. Rejeski (Ph.D.), Dr. G. Goldberg (Ph.D.), Dr. A. Khadilkar (M.D.),
Dr. L. Pelland (Ph.D.) and Ms. G. Paterson for their valuable comments concerning the initial proposal.

This study was completed with the support of a research grant obtained from the Canadian Institutes of Health Research (CIHR) ( Grant\#MCT82367); University Research Chair (salary support for research staff) and the Ministry of Human Resources (summer student program) (Canada). This RCT won a prize for the best community-based project from the City of Gatineau (Canada) in 2009. This RCT contributed to enhance the quality of life of individuals with mild and moderate OA of the knee in Canada's National Capital region.

\section{Author details}

${ }^{1}$ Public Health, specialization in Epidemiology, University Research Chair, School of Rehabilitation Sciences, University of Ottawa, Ottawa, Canada. ${ }^{2}$ Epidemiology and Biostatistics, Department of Epidemiology and Community Medicine, University of Ottawa, Ottawa, Canada. ${ }^{3}$ School of Human Kinetics, Faculty of Health Sciences, University of Ottawa, Ottawa, Ontario, Canada. ${ }^{4}$ University of Ottawa Heart Institute, Ottawa, Ontario, Canada. ${ }^{5}$ University of Toronto, Toronto, Toronto, Canada. ${ }^{6}$ Epidemiology, Chairman, Centre for Global Health, Institute of Population Health, University of Ottawa, Ottawa, Canada. ${ }^{7}$ Baycrest Centre, Toronto, Canada. ${ }^{8}$ Inter-Action Rehabilitation Inc., Toronto, Ontario, Canada. ${ }^{9}$ Department of Epidemiology and Community Medicine, University of Ottawa, Ottawa, Canada. ${ }^{10}$ University of Ottawa Heart Institute, Ottawa, Ontario, Canada.

Received: 3 February 2012 Accepted: 29 August 2012

Published: 12 December 2012

\section{References}

1. Felson DT, Zhang Y: An update on the epidemiology of knee and hip osteoarthritis with a view to prevention. Arthritis Rheum 1998, 41(8):1343-1355.

2. Slemenda CW: The epidemiology of osteoarthrtis of the knee. Curr Opin Rheumatol 1992, 4:546-451.

3. Brosseau L, Wells G, Tugwell P, Egan M, Dubouloz CJ, Casimiro L, Robinson V, Pelland L, McGowan J, Lamb M: Ottawa Panel evidence-based clinical practice guidelines for therapeutic exercises and manual therapy in the treatment of osteoarthritis. Phys Ther 2005, 85(9):907-971.

4. Loew L, Brosseau L, Wells G, Tugwell P, Egan M, Dubouloz CJ, Casimiro L, Welch V, McEwan J: Ottawa Panel evidence-based clinical practice guidelines for walking programs in the treatment of osteoarthritis. Arch Phys Med Rehabil 2012, 93(7):1269-1285.

5. Brosseau L, Wells GA, Tugwell P, Egan M, Dubouloz CJ, Casimiro L, Bugnariu N, Welch V, De Angelis G, Francoeur L, Milne S, Loew L, McEwan J, Messier SP, Doucet E, Kenny GP, Prud'homme D, Lineker S, Bell M, Poitras S, Li JX, Finestone HM, Laferrière L, Haines-Wangda A, Russell-Doreleyers M, Lambert K, Marshall AD, Cartizzone M, Teav A: Ottawa Panel evidence-based clinical practice guidelines for the management of osteoarthritis in adults who are obese and overweight. Phys Ther 2011, 91(6):843-861.

6. Brosseau L, Wells GA, Kenny GP, Reid R, Maetzel A, Tugwell P, Huijbregts M, McCullough C, De Angelis G, Chen L: The implementation of a community-based aerobic walking program for mild to moderate knee osteoarthritis (OA): a knowledge translation (KT) randomized controlled trial (RCT): Part I: The Uptake of the Ottawa Panel clinical practice guidelines (CPGs). BMC Public Health 2012, 12:871.

7. Graham ID, Logan J, Harrison MB, Straus SE, Tetroe J, Caswell W, Robinson $\mathrm{N}$ : Lost in knowledge translation: time for a map? J Contin Educ Health Prof 2006, 26(1):13-24.

8. Straus SE, Tetroe J, Graham ID: Knowledge translation in health care: moving from evidence to practice. Chichester: Wiley-Blackwell/BMJ; 2009:318.

9. Messier SP, Loeser RF, Mitchell MN, Valle G, Morgan TP, Rejeski WJ, Ettinger WH: Exercise and weight loss in obese older adults with knee osteoarthritis: a preliminary study. J Am Geriatric Soc 2000, 48(9):1062-1072.

10. Huang MH, Chen $\mathrm{CH}$, Chen TW, Weng MC, Wang WT, Wang YL: The effects of weight reduction on the rehabilitation of patients with knee osteoarthritis and obesity. Arthritis Care Res 2000, 13(6):398-405.

11. Sniezek JE, Macera CA, Hootman JM, Eyler AA: Work Group Recommendations: 2002 Exercise and Physical Activity Conference - Session II: the problem and challenge of inactivity, St Louis, Missouri. Arthritis Rheum 2003, 49(3):453-454. 
12. Macera CA, Hootman JM, Sniezek JE: Major public health benefits of physical activity. Arthritis Rheum 2003, 49(1):122-128.

13. Hootman JM, Macera CA, Ham SA, Helmick CG, Sniezek JE: Physical activity levels among the general US adult population and in adults with and without arthritis. Arthritis Rheum 2003, 49(1):129-135.

14. Sharpe PA: Community-based physical activity intervention. Arthritis Rheum 2003, 49(3):455-462.

15. Minor M, Stenström $\mathrm{CH}$, Klepper SE, Hurley M, Ettinger WH: Work Group Recommendations: 2002 Exercise and Physical Activity Conference -Session V: evidence of benefit of exercise and physical activity in arthritis, St. Louis, Missouri. Arthritis Rheum 2003, 49(3):453-454.

16. Chang R, Roubenoff R, Mayer J: Work Group Recommendations: 2002 Exercise and Physical Activity Conference - Session IV: exercise in the presence of rheumatic disease, St. Louis, Missouri. Arth Rheum (Arthritis Care Res) 2003, 49(2):280

17. Brady TJ, Sniezek JE: Implementing the national arthritis action plan: New population-based approaches to increasing physical activity among people with arthritis. Arthritis \& Rheum (Arthritis Care Res) 2003, 49(3):471-476.

18. Bautch JC, Malone DG, Vailas AC: Effects of exercise on knee joints with osteoarthritis: A pilot study of biological markers. Arthritis Care Res 1997, 10(1):48-55

19. Fransen $M$, Crosbie J, Edmonds J: Physical therapy is effective for patients with osteoarthritis of the knee: A randomized controlled clinical trial. $J$ Rheumatol 2001, 28(1):156-164.

20. Minor MA, Hewett JE, Webel RR, Anderson SK, Kay DR: Efficacy of physical conditioning exercise in rheumatoid arthritis and osteoarthritis. Arthritis Rheum 1989, 32(11):1396-1405.

21. O'Reilly SC, Muir KR, Doherty M: Effectiveness of home exercise on pain and disability from osteoarthritis of the knee: a randomised controlled trial. Ann Rheum Dis 1999, 58(1):15-19.

22. Peterson MG, Kovar-Toledano PA, Otis JC, Allegrante JP, Mackenzie CR, Gutin B, Kroll MA: Effect of a Walking Program on Gait Characteristics in Patients with Osteoarthritis. Arthritis Care Res 1993, 6(1):11-16.

23. Sullivan T, Allegrante JP, Peterson MG, Kovar PA, Mackenzie CR: One-year follow up of patients with osteoarthritis of the knee who participated in a program of supervised fitness walking and supportive patient education. Arthritis Care Res 1998, 11(4):228-233.

24. Dias RC, Dias JM, Ramos LR: Impact of an exercise and walking protocol on quality of life for elderly people with OA of the knee. Physiother Res Int 2003, 8(3):121-130.

25. Minor MA, Brown JD: Exercise maintenance of persons with arthritis after participation in a class experience. Health Educ Q 1993, 20(1):83-95.

26. Hurley MV: Muscle dysfunction and effective rehabilitation of knee osteoarthritis outcomes: What we need to find out. Arthritis Rheum 2003, 49(3):444-452

27. Haskell WL: The efficacy and safety of exercise programs in cardiac rehabilitation. Med Sci Sports and Exercise 1994, 26(7):815-823.

28. DeBusk RF, Miller NH, Superko HR, Dennis CA, Thomas RJ, Lew HT, Berger WE 3rd, Heller RS, Rompf J, Gee D, Kraemer HC, Bandura A, Ghandour G, Clark M, Shah RV, Fisher L, Taylor CB: A case-management system for coronary risk factor modification after acute myocardial infarction. Ann Intern Med 1994, 120(9):721-729.

29. Kirk S: Diet and weight management. Nurs Stand 2003, 17(49):47-53.

30. McKay HG, King D, Eakin EG, Seeley JR, Glasgow RE: The diabetes network internet-based physical activity intervention: a randomized pilot study. Diabetes Care 2001, 24(8):1328-1334.

31. Munneke M, De Jong Z, Zqinderman AH, Jansen A, Ronday HK, Pter WF, Boonman DC, van den Ende CH, Vliet Vlieland TP, Hazes JM: Adherence and satisfaction of rheumatoid arthritis patients with a long-term intensive dynamic exercise program (RAPIT program). Arthritis Rhuem 2003, 49(5):665-672.

32. Van Tulder MW, Assendett WJ, Koes BW, Bouter LM: Method guidelines for systematic reviews in the Cochrane Collaboration Back Review Group for spinal disorders: Operationalization of Van Tulder's quality assessment form. Spine 1997, 22(20):2323-2330.

33. Jadad AR, Moore RA, Carroll D, Jenkinson C, Reynolds DJ, Gavaghan DJ, McQuay HJ: Assessing the Quality of Reports of Randomized Clinical Trials: Is Blinding Necessary? Control Clin Trials 1996, 17(1):1-12.

34. Bombardier C, Melfi CA, Paul J, Green R, Hawker G, Wright J, Coyte P: Comparison of a generic and a disease specific measure of pain and physical function after a knee replacement surgery. Arthritis Rheum 1994, 37:S225.

35. Stucki G, Liang MH, Phillips C, Katz JN: The short form-36 is superior to sickness impact profile as a generic health status measure in patients ongoing total hip arthoplasty. Arthritis Rheum 1995, 8(3):174-181.

36. Carr A: Adult Measures of Quality of Life: The Arthritis Impact Measurement Scales (AIMS/AIMS2), Disease Repercussion Profile (DRP), EuroQoL, Nottingham Health Profile (NHP), Patient Generated Index (PGI), Quality of Well-Being Scale (QWB), RAQoL, Short Form-36 (SF-36), Sickness Impact Profile (SIP), SIP-RA, and World Health Organization's Quality of Life Instruments (WHOQoL, WHOQoL-100, WHOQoL-Bref). Arthritis Rheum (Arthritis Care \& Res.) 2003, 49:S113-S133.

37. Ware JE: SF-36 Physical and mental health summary scales: a user's manual. Boston: The health institute; 1994.

38. Ware JE Jr, Sherbourne CD: The MOS 36-item short-form health survey (SF-36) 1. A conceptual framework and item selection. Med Care 1992, 30(6):473-483.

39. Wilson AS, Kitas GD, Carruthers DM, Reay C, Skan J, Harris S, Treharne GJ, Young SP, Bacon PA: Computerized information-gathering in specialist rehumatology clinics: an initial evaluation of an electronic version of the Short-Form 36. Rheumatology 2002, 41(3):268-273.

40. Kosinski M, Keller SD, Hatoum HT, Kong SX, Ware JE: The SF-36 health survey as a generic outcome measure in clinical trials of patients with osteoarthritis and rheumatoid arthritis: tests of data quality, scaling assumptions, and score reliability. Med Care 1999, 37:M10-M22.

41. Ruta DA, Hurst NP, Kind P, Hunter M, Stubbings A: Measuring health status in British patients with rheumatoid arthritis: reliability, validity and responsiveness of short-form 36-items health survey (SF-36). Br J Rheumatol 1998, 37(4):425-436.

42. Hays RD, Sherbroune CD, Mazel RM: The RAND 36-item Health Survey 1.0. Health Econ 1993, 2(3):217-227.

43. Meenan RF, Gertman PM, Mason JH: Measuring health status in arthritis: the Arthritis Impact Measurements Scales. Arthritis Rheum 1980, 23(2):146-152

44. Meenan RF, Mason JH, Anderson JJ, Guccione AA, Kazis LE: AIM2: the content and properties of a revised and expanded Arthritis Impact Measurement Scales health status questionnaire. Arthritis Rheum 1992, 35(1):1-10.

45. Bellamy N, Buchanan WW: A preliminary evaluation of the dimensionality and clinical importance of pain and disability in osteoarthritis of the knee and hip. Clin Rheumatol 1986, 5(2):231-241.

46. Bellamy N, Buchanan WW, Golsmith CH, Campbell J, Stitt LW: Validation study of WOMAC: a health status instrument for measuring clinically important patients relevant, outcomes to antirheumatic drug therapy in patients with OA of the hip or knee. J Rheumatol 1988, 15(12):1833-40

47. Evcik D, Sonel D: Effectiveness of a home-based exercise therapy and walking program on osteoarthritis of the Knee. Rheumatol Int 2002, 22(3):103-106.

48. Halbert J, Crotty M, Weller D, Ahern M, Silagy C: Primary care-based physical activity programs: Effectiveness in sedentary older patients with osteoarthritis symptoms. Arthritis Rheum 2001, 45(3):228-234.

49. McConnell S, Kolopack P, Davis AM: The Western Ontario and Mc Master Universities Osteoarthritis Index (WOMAC): a review of its utility and measurement properties. Arthritis Rheum 2001, 45(5):453-561.

50. Rogers JC, Irrgang JJ: Measures of adult lower extremity function. Arthritis Care Res 2003, 49(5S):S67-S84.

51. Angst F, Aeschlimann A, Steiner W, Stucki G: Responsiveness of the WOMAC osteoarthritis index as compared with the SF-36 in patients with osteoarthritis of the legs undergoing a comprehensive rehabilitation intervention. Annals Rheum Dis 2001, 60(9):834-840.

52. Kovar PA, Allegrante JP, Mackenzie CR, Peterson MG, Gutin B, Charlson ME: Supervised fitness walking in patients with osteoarthritis of the knee. A randomized, controlled trial. Ann Intern Med 1992, 116(7):529-534.

53. Messier SP, Thompson CD, Ettinger MH: Effects of long-term aerobic or weight training regimens on gait in an older, osteoarthritic population. J Appl Biomech 1997, 13:202-225.

54. Hughes SL, Seymour RB, Campbell R, Pollak N, Huber G, Sharma L: Impact of the fit and strong intervention on older adults with osteoarthritits. Gerontologist 2004, 44(2):217-228.

55. Talbot LA, Gaines JM, Huynh TN, Metter EJ: A home-based pedometer-driven walking program to increase physical activity in older 
adults with osteoarthritis of the knee: A preliminary study. J Am Geriatric Soc 2003, 51(3):387-392.

56. Podsiadlo D, Richardson S: The timed "Up \& Go": a test of basic functional mobility for frail elderly persons. J Am Geriatr Soc 1991, 39(2):142-148.

57. Sallis JF, Buono MJ, Roby JJ, Micale FG, Nelson JA: Seven-Day Physical Activity Recall. Med Sci Sports Exerc 1997, 29:89-103.

58. Hayden-Wade HA, Coleman KJ, Sallis JF, Armstrong C: Validation of the telephone and in-person interview versions of the 7-day PAR. Med SC Sports Exerc 2003, 35(5):801-809.

59. Miller GD, Nicklas BJ, Davis C, Loeser RF, Lenchik L, Messier SP: Intensive Weight Loss Program Improves Physical Function in Older Obese Adults with Knee Osteoarthritis. Obesity (Silver Spring) 2006, 14(7):1219-1230.

60. Talbot LA, Gaines JM, Ling SM, Metter EJ: A home-based protocol of electrical muscle stimulation for quadriceps muscle strength in older adults with osteoarthritis of the knee. J Rheumatol 2003, 30(7):1571-1578.

61. Wang X, Miller GD, Messier SP, Nicklas BJ: Knee Strength Maintained Despite Loss of Lean Body Mass During Weight Loss in Older Obese Adults With Knee Osteoarthritis. J Gerontology 2007, 62A(8):866-870.

62. Messier SP, Loeser RF, Miller GD, Morgan TM, Rejeski WJ, Sevick MA, Ettinger WH Jr, Pahor M, Williamson JD: Exercise and dietary weight loss in overweight and obese older adults with knee osteoarthritis. The arthritis, diet and activity promotion trial. Arthritis Rheum 2004, 50(5):1501-1510.

63. Focht BC, Rejeski J, Ambrosius WT, Katula JA, Messier SP: Exercise, Self-Efficacy, and Mobility Performance in Overweight and Obese Older Adults with Knee Osteoarthritis. Arthritis Rheum 2005, 53(5):659-665.

64. Penninx BW, Messier SP, Rejeski WJ, Williamson JD, Dibari M, Cavazzini C, Applegate WB, Pahor M: Physical exercise and the prevention of disability in activities of daily living in older persons with osteoarthritis. Arch Intern Med 2001, 161(19):2309-2316.

65. Toda Y: The effect of energy restriction, walking, and exercise on lower extremity lean body mass in obese women with osteoarthritis of the knee. J Orthopedic Sci 2001, 6(2):148-154.

66. Tak E, Staats P, van Hespen A, Hopman-Rock M: The Effects of an Exercise Program for Older Adults with Osteoarthritis of the Hip. J Rheumatol 2005, 32(6):1106-1113.

67. Peloquin L, Bravo G, Gauthier P, Lacombe G, Billiard JS: Effects of a cross-training exercise program in persons with osteoarthritis of the knee. A randomized controlled trial. J Clin Rheumatol 1999, 5:126-136.

68. Bement MKH, Weyer A, Hartley S, Yoon T, Hunter SK: Fatiguing exercise attenuates pain-induced corticomotor excitability. Neurosci Lett 2009, 452(2):209-213.

69. Stagg NJ, Mata HP, Ibrahim MM, Henriksen EJ, Porreca F, Vanderah TW, Malan PT: Regular Exercise Reverses Sensory Hypersensitivity in a Rat Neuropathic Pain Model. Anesthesiology 2011, 14(4):940-948.

70. Koltyn KF: Analgesia following exercise: A review. Sports Med 2000, 29(2):85-98.

71. Garfinkel MS, Schumacher HR Jr, Husain A, Levy M, Reshetar RA: Evaluation of a yoga based regimen for treatment of osteoarthritis of the hands. J Rheumatol 1994, 21(12):2341-2343.

72. Thorstensson CA, Henriksson M, von Porat A, Sjodahl C, Roos EM: Eight weeks of exercise reduced knee adduction moment during one-leg rise in patients with early knee osteoarthritis. Disabil Rehabil 2005, 2:1-11.

\section{doi:10.1186/1471-2458-12-1073}

Cite this article as: Brosseau et al:: The implementation of a communitybased aerobic walking program for mild to moderate knee osteoarthritis: A knowledge translation randomized controlled trial: Part II: Clinical outcomes. BMC Public Health 2012 12:1073.

\section{Submit your next manuscript to BioMed Central and take full advantage of:}

- Convenient online submission

- Thorough peer review

- No space constraints or color figure charges

- Immediate publication on acceptance

- Inclusion in PubMed, CAS, Scopus and Google Scholar

- Research which is freely available for redistribution 